\title{
RACIONALIDAD Y PROGRESO DEL DESARROLLO CIENTÍFICO: UNA CONTROVERSIA METAMETODOLÓGICA
}

LEÓN OLIVÉ

INSTTIUTO DE INVESTIGACIONES FILOSÓFICAS

UNAM

En este trabajo me propongo examinar la relación entre las nociones de racionalidad y progreso, aplicadas al problema del desarrollo científico. Para ello partiré de la consideración de un enunciado metametodológico que, además de involucrar un análisis de la relación entre los conceptos mencionados, conduce también a problemas como los de la estructura y el proceso de cambio de los marcos conceptuales en la ciencia y el papel de la historia de la ciencia en la evolución de metodologías científicas y, en general, su relación con la filosofía de la ciencia.

El enunciado metametodológico en cuestión tiene la forma de un imperativo y puede expresarse como una norma metametodológica:

(NMF) Norma metametodológica fundamental:

Una metodología científica debe reconstruir lo más posible de la ciencia del pasado como una actividad racional que produce resultados bien fundados y que se desarrolla racionalmente.

De esta norma fundamental se deriva un criterio comparativo para la elección entre metodologías:

(CEM) Criterio de elección entre metodologías:

Una metodología de la ciencia es preferible a otra si reconstruye como racionales más episodios de la historia de la ciencia que la otra y si muestra que esta historia reconstruida es progresiva.

Podrían surgir de inmediato dos preocupaciones con respecto a esta norma y este criterio. Una sería relativa a su vaguedad e imprecisión. La otra a que efectivamente sean, o hayan sido, significativamente defendidos dentro de la filosofía de la ciencia contemporánea. Sobre el primer problema me extenderé más adelante, precisamente con la intención de precisar su formulación para luego evaluar su importancia dentro de la filosofía de la ciencia. Con respecto 
a la segunda preocupación, puede recordarse por ejemplo la idea de Lakatos de que

todas las metodologías funcionan como teorías (o programas de investigación) historiográficas (o metahistóricas) y pueden ser criticadas mediante la crírica de las reconstrucciones históricas racionales que originan. (Lakatos, 1978, p. 121)

\section{Metodología}

Para analizar la norma metametodológica (NMF) y el criterio derivado (CEM), es necesario, en primer lugar, indicar en qué sentido se entiende el término 'metodología'. Por una metodología entenderé un modelo de cambio y desarrollo científicos. Este modelo debe incluir normas que se aplican dentro de los contextos científicos propiamente dichos, como por ejemplo, según ciertas metodologías, que los científicos deberían hacer generalizaciones inductivas o, según otras, que los científicos deberían aceptar sólo aquellas proposiciones que sean falsables empíricamente. Diferentes metodologías pueden proponer normas distintas, lo cual puede conducir a rivalidades entre las metodologías. Las normas del tipo aludido forman parte de los criterios de cientificidad y de los criterios de evaluación de teorías científicas, los cuales deberían estar también incluidos en las metodologías. Tales normas forman parte de esos criterios en el sentido de que las acciones o las decisiones de los científicos deberían mostrarse conformes con ellas para ser aceptadas efectivamente como científicas y como conducentes a genuino conocimiento científico. Las metodologías también incluyen valores, de acuerdo con los cuales se orientan las acciones de los científicos y los cuales operan también para la elección de teorías. Asimismo, las metodologías incluyen los fines en función de los cuales se produce, evalúa y acepta el conocimento científico (de acuerdo con cada metodología). Sin embargo, una cierta metodología puede reconocer que no existe un único conjunto de normas y reglas de investigación científica que sean válidas en todo momento del desarrollo de la ciencia; en diferentes épocas pueden estar vigentes diferentes normas. Lo mismo ocurre con los fines de la investigación científica.

Una metodología, entendida así, presupone una cierta concepción epistemológica de la ciencia, es decir, una concepción de la naturaleza y de la justificación del conocimiento científico, incluyendo una concepción del papel de las acciones de los científicus en la producción, evaluación y aceptación de ciertos cuerpos de creencias como genuino conocimiento científico.

Tal vez puede entenderse mejor lo anterior si subrayamos los siguientes niveles en las discusiones de filosofía de la ciencia:

1) El primer nivel es el de los análisis en cada ciencia particular, especialmente de sus teorías y métodos específicos; éstos incluyen normas, en parti- 
cular reglas de experimentación y criterios de evaluación para la aceptación de creencias como conocimento científico dentro del campo en cuestión.

2) El segundo nivel es el de las metodologías en el sentido arriba indicado. Los trabajos más influyentes en la filosofía de la ciencia de los últimos treinta años, por ejemplo los de Popper, Kuhn, Lakatos, Laudan y Shapere, pueden interpretarse como si ofrecieran modelos de desarrollo científico, lo cual quiere decir que reconstruyen la metodología científica, en el sentido aquí indicado. Para reconstruir adecuadamente el proceso de desarrollo científico, los modelos deben incluir, aunque sea en la forma de presupuestos, concepciones acerca de la estructura de las teorías científicas.

3) El tercer nivel contiene explícitamente las concepciones epistemológicas acerca de las ciencias, las cuales pueden estar sólo presupuestas en los modelos de desarrollo. Estas son las concepciones acerca de la naturaleza del conocimiento científico y del problema de su justificación y aceptabilidad racional; incluyen ideas sobre el papel de la observación y la experimentación en las ciencias, así como acerca de los marcos conceptuales presupuestos por las teorías y las actividades científicas. Contienen también ideas sobre la objetividad, la racionalidad y el progreso científicos. Se trata, pues, del nivel en el que se someten a discusión y crítica las metodologías mismas, entendidas como modelos de desarrollo científico; por esta razón puede llamársele legítimamente el nivel de la metametodología, y las disputas dentro de este nivel pueden entenderse como controversias metametodológicas.

\section{Racionalidad}

Un térmiro clave en la formulación de NMF es el de 'racionalidad'. Sin embargo, existen muchos sentidos posibles de este término, lo cual da lugar a una seria ambigüedad. En el caso de la ciencia del pasado, el término 'racionalidad' suele aplicarse a por lo menos dos tipos diferentes de cosas: por un lado a las decisiones y actividades de los científicos, y por extensión a las creencias que se generan y aceptan como resultados de tales decisiones y acciones; por otro lado el término se aplica al proceso de desarrollo de la ciencia.

A su vez las decisiones de los cientíticos pueden referirse a:

a) medios para obtener fines dados;

b) valores y fines;

c) aceptación o rechazo de concepciones del mundo (entramados conceptuales más comprensivos que las teorías);

d) aceptación o rechazo de teorías;

e) aceptación o rechazo de datos como evidencia pertinente;

f) aceptación de reglas y normas; 
g) aceptación o rechazo de principios lógicos.

Cada uno de estos niveles da lugar a un concepto de racionalidad, y coinciden en gran medida con los conceptos de racionalidad que Mario Bunge ha planteado explícitamente en un artículo titulado "Seven Desiderata for Rationality" (Bunge, 1987). Siguiendo de mancra aproximada sus ideas, cada uno de los anteriores niveles puede verse como si diera lugar a los siguientes conceptos de racionalidad:

a) Racionalidad instrumental o de medios-fines: trata de la elección de los medios más adecuados para la obtención de fines dados.

b) Racionalidad evaluativa: acerca de los fines que son realizables y que merecen ser perseguidos porque les asignamos un valor.

c) Racionalidad ontológica: requiere la adopción de una visión del mundo consistente, compatible con la ciencia y la tecnología disponibles.

d) Racionalidad epistemológica: requiere que las teorías que se acepten tengan apoyo empírico y evita conjeturas que sean incompatibles con el cuerpo de conocimiento científico y tecnológico aceptado por medio de buenas razones.

e) y f) Racionalidad metodológica: requiere que la aceptación de creencias se haga después de una crítica cuidadosa y sólo cuando exista una adecuada justificación, es decir, pruebas o evidencia favorable y que no existan rażones suficientemente poderosas como para abandonar la creencia en cuestión; asimismo exige que la aceptación de creencias esté basada en normas y reglas que tengan alguna garantía, aunque también ellas sean revisables.

g) Racionalidad lógica: exige evitar las contradicciones.

Puede llamarse racionalidad completa al concepto que englobe todos estos niveles. Cuando se ejemplifica uno de estos niveles, o una combinación de algunos de ellos, podemos hablar de racionalidad parcial.

La anterior caracterización de la racionalidad completa pretende tener utilidad para el análisis de la racionalidad y el progreso de la ciencia, no pretende caracterizar algún concepto universal de racionalidad.

Al hablar de la racionalidad de los científicos al tomar ciertas decisiones o al realizar cierto tipo de acciones debe distinguirse entre la racionalidad de los científicos como individuos y la racionalidad de las comunidades. Por ejemplo, puede resultar racional para una comunidad el que diferentes subgrupos de ella persigan programas diferentes, aunque analizadas a nivel individual, o a nivel de los subgrupos, y dejando de lado el contexto de toda la comunidad, algunas de las decisiones de perseguir tal o cual programa a la larga parezcan irracionales; sin embargo, analizadas en el contexto de la comunidad, es decir, al tomar en cuenta los recursos, las creencias y el conocimiento previo 
disponibles para esa comunidad, esas acciones pueden resultar racionales, por ejemplo bajo un principio de cooperación dentro de la comunidad, o bajo un principio según el cual la comunidad queda "cubierta" en las apuestas que hace (véase, por ejemplo, Wykstra, 1980).

Conviene señalar que en el caso de la racionalidad instrumental, la toma de decisiones o las acciones que se ejecutan requieren un acuerdo acerca de los principios lógicos, normas, valores, ejemplos paradigmáticos, creencias y conocimientos previos y fines, esto es, las decisiones se toman bajo el supuesto de que se comparte un mismo marco conceptual.

En cuanto a las aplicaciones del predicado 'racional' al desarrollo de la ciencia conviene distinguir claramente la racionalidad del proceso de desarrollo científico mismo y la toma de decisiones de los científicos o las comunidades en cada uno de los niveles antes mencionados. Ese proceso debe verse como una sucesión de marcos conceptuales dentro de los cuales existen principios lógicos, normas y reglas, métodos de investigación, conocimientos y creencias, teorías, valores y fines. Daré por sentado que la filosofía de la ciencia postempirista ha ofrecido suficientes razones para considerar que el desarrollo de la ciencia está constituido por esa sucesión de marcos conceptuales.

Bajo este planteamiento existen dos problemas de interés:

Primero: ¿qué puede querer decir que dicho proceso de desarrollo científico sea racional?

Segundo: ¿puede reducirse la racionalidad del proceso mismo a las decisiones y acciones de los científicos o de las comunidades de los científicos?, es decir, la calificación de racional del proceso éno significa nada más que ser el resultado de decisiones y acciones de los científicos o de las comunidades, las cuales pueden calificarse de racionales? Es claro que ese proceso es resultado, en parte, del conjunto de decisiones y acciones realizadas por los científicos, pero ise agota la explicación y el significado mismo de lo que quiere decir que el proceso de desarrollo científico sea racional con las decisiones de los científicos o de las comunidades? Lo que me propongo defender adelante es que la respuesta debe ser negativa: el proceso de desarrollo científico, tal y como puede ser legítimamente reconstruido, tiene una genuina autonomía con respecto a las decisiones de los agentes, sean científicos individuales o sean comunidades.

Para esto es necesario insistir en que el proceso de desarrollo científico consiste en una sucesión de marcos conceptuales. Así, la noción de racionalidad del proceso de desarrollo puede plantearse en términos de la racionalidad de las transformaciones de los sucesivos marcos conceptuales: $\operatorname{sean} M C_{1}$ y $M C_{2}$ dos marcos conceptuales sucesivos en el proceso de desarrollo científico (de acuerdo con una cierta reconstrucción), la transformación del primero en el segundo, o la sustitución del primero por el segundo, es racional si un sujeto 
racional que parta de $M C_{1}$ puede ser convencido, en una discusión racional con un sujeto racional que se adhiera a $M C_{2}$, de realizar transformaciones en $M C_{1} \multimap$ de abandonarlo- de tal manera que las transformaciones resulten en $M C_{2}$.

Esto presupone varias ideas:

1) Que los agentes que se adhieran a uno y otro marco conceptual tienen una competencia comunicativa y una capacidad de diálogo.

2) La posibilidad de que dicha capacidad pueda ejercerse discutiendo únicamente en términos de razones y excluyendo ejercicios de poder que no sean epistémicos, es decir, que no se basen en la fuerza de las razones, de las pruebas $\mathrm{y}$ de los argumentos.

3) Que a partir de la realización de los elementos que suponen 1) y 2) puedan ponerse a discusión, entre agentes que parten de marcos distintos, los principios lógicos aceptados dentro de cada marco, así como sus respectivas reglas y normas metodológicas, conocimientos y creencias previos, concepciones del mundo, valores y los fines, así como los medios que se consideran adecuados para obtener los fines buscados. Cada uno de estos elementos puede ponerse a discusión de manera individual y en la discusión puede recurrirse a todos los otros niveles. Así, por ejemplo, puede pensarse en la discusión de un cierto fin que persiga el sujeto que se adhiere a $M C_{1}$; tal vez su marco conceptual le impide darse cuenta de que el fin en cuestión es irrealizable, nuestro supuesto es que es posible aducir razones que provienen de $M C_{2}$ y que pueden resultar aceptables para ese sujeto precisamente como razones para reconocer que el fin que perseguía es irrealizable (aunque tal vez requiera cambios en $M C_{1}$, pero cambios que gradualmente puede aceptar hacer, también con base en razones). Todo esto supone que puede haber modificaciones importantes en $M C_{1}$, además de que el resultado de la discusión sea el abandono del fin en cuestión. Los cambios en los presupuestos bajo los cuales piensa y actúa el sujeto en cuestión (principios lógicos, normas, creencias previas, etc.) pueden llegar a ser tan fundamentales que ya no coincidan más con $M C_{1}$, sino que se acerquen más a $M C_{2}$. Esta es la idea central dentro de la concepción de cambios graduales y racionales de marcos conceptuales. Pensemos en otro ejemplo, digamos en la modificación de la idea acerca del dominio de aplicación de un cierto principio lógico, por ejemplo, que se cambie de la idea de que tenía un dominio universal de aplicación, a la idea de que existen razonamientos sobre los cuales no es aplicable; esto puede ocurrir, digamos, si se aceptan ciertas teorías sustantivas, cierta visión del mundo, por ejemplo la que se deriva de la aceptación de la mecánica cuántica, pues entonces puede pensarse que un sujeto racional que admita la mecánica cuántica podría ser llevado por medio de una discusión racional a la modificación de su marco conceptual, en el sentido de reconocer que para razonar acerca del nivel de los fenómenos cuánticos no se aplican todos los principios de la lógica clásica. 
Análogamente, una cierta visión del mundo preferida bajo un cierto marco conceptual, puede llegar a ser modificada racionalmente por los agentes racionales que se adhieran a ella, si en una discusión racional se les muestra que su visión del mundo es internamente inconsistente, inconsistencia que quizá desde su marco conceptual fuera inapreciable, pero que a la luz de evidencia disponible a partir de otro marco conceptual puede quedar en claro.

Lo anterior quiere decir que en el proceso de desarrollo de la ciencia es posible reconstruir las transformaciones de los marcos conceptuales de una manera en la cual pueden encontrarse razones que para los científicos y las comunidades en cuestión hubieran sido suficientes para modificar elementos de sus marcos conceptuales, desde principios lógicos hasta normas, valores y fines. El compromiso de la reconstrucción es que esas razones efectivamente hubieran sido razones comprensibles y aceptables para los científicos o las comunidades de que se trata, aunque eso hubiera requerido una discusión racional que quizá no se haya dado de hecho como fenómeno histórico.

Mediante el examen de este tipo de razones es posible hablar de una reconstrucción racional del proceso de desarrollo de los marcos conceptuales presupuestos por las teorías y las actividades científicas. Según lo expuesto, la transformación de un marco conceptual en otro sucesivo puede reconstruirse como racional, y por eso todo el proceso puede reconstruirse como racional, aunque marcos conceptuales que se encuentren bastante alejados entre sí puedan diferir en casi todos sus elementos, o incluso en todos.

Notemos que la idea anterior rechaza la noción de racionalidad metodológica como conjunto de reglas y normas válidas para toda comunidad y dentro de todo marco conceptual para justificar la adopción de ciertas creencias o la ejecución de ciertas acciones. Los conjuntos de reglas pueden estar fijos dentro de cada marco conceptual, pero pueden variar de uno a otro. Por esto, la racionalidad del proceso de desarrollo científico debe entenderse en el sentido de racionalidad completa, según el sentido sugerido arriba. Esto quiere decir que el proceso admite una reconstrucción según la cual se presupone esa racionalidad completa, la cual funciona como idea regulativa, pero que se ejemplifica de hecho, al menos como racionalidad parcial, en numerosas ocasiones dentro del proceso. Esa idea implica también que los científicos son sujetos que pueden ejercer su capacidad de ser racionales, la cual aquí se entiende en el sentido de una disposición a actuar y a sostener creencias, valores y fines de acuerdo con la racionalidad completa.

Deliberadamente he hablado de la reconstrucción racional del proceso de desarrollo de la ciencia y no de la historia de la ciencia. La reconstrucción racional se refiere a la reconstrucción de los marcos conceptuales y a su sucesión. Puede haber reconstrucciones diferentes, cada una de las cuales conduce a un cierto modelo de desarrollo, que es lo que he llamado una metodología. Es preciso distinguir entre las reconstrucciones racionales y la cuestión que de becho 
los científicos o las comunidades hayan modificado sus marcos, o los hayan sustituido, mediante decisiones tomadas sobre la base de las razones que a posteriori podemos reconstruir. Quizá en ocasiones la sustitución de un marco por otro se debió a causas no epistémicas, por ejemplo ejercicios de poder u otro tipo de factores sociales no epistémicos, tales como influencias de tipo ideológico, coerción, etc. Eso no sería un obstáculo, en principio, para realizar una reconstrucción del estilo de la sugerida que sea racional y correcta en el sentido de que, independientemente de la cuestión de hecho, las razones que pueden observarse en la reconstrucción hubieran sido accesibles a los agentes pertinentes. Esta es la razón por la cual la noción de racionalidad del proceso de desarmllo cientifico no puede reducirse a la toma de decisiones racionales por parte de los científicos individuales o de las comunidades.

\section{Racionalidad y progreso}

Para ubicar la importancia de las consideraciones anteriores en el contexto de las actuales discusiones metametodológicas y para subrayar la conexión conceptual entre racionalidad y progreso en el desarrollo científico, contrastaré las ideas aquí expresadas con un trabajo de Laudan (1987), en donde este autor sostiene que la única noción de racionalidad que importa en el caso del desarrollo científico es la de racionalidad instrumental, la racionalidad de medios-fines. Más aún, Laudan sostiene que una correcta filosofía de la ciencia requiere de una noción de progreso, pero puede prescindir de la de racionalidad en sus reconstrucciones del desarrollo científico. Objetaré esta conclusión con base en los análisis de la noción de racionalidad que he sugerido.

Laudan sostiene que todos los intentos de reconstrucción racional del desarrollo científico comparten el supuesto de que si las ciencias se han desarrollado racionalmente y han producido resultados (teorías) bien fundados, entonces una metodología de la ciencia debe ser, 20 ipso, una teoría de la racionalidad. La cita de Lakatos reproducida arriba parece prestar apoyo también a esta interpretación.

Más aún, bajo una interpretación como la que sugiere Laudan - para luego criticarla- el análisis de la racionalidad científica, tal como se realizaría con base en una cierta metodología, presupone efectivamente que la ciencia ha sido racional tanto en el sentido de que los científicos han tomado decisiones racionales, como de que el proceso ha sido racional. Adelante insistiré en que puede ser el caso que se reconstruya el proceso de desarrollo científico como racional, sin que eso implique que necesariamente todas las decisiones de los científicos que hayan sido pertinentes para ese desarrollo hayan sido decisiones racionales.

Laudan ha sostenido (1986 y 1987) que las posiciones que admiten algo como la norma metametodológica fundamental y su criterio de comparación 
asociado - mencionados al principio de este trabajo- y que consideran que las metodologías pueden ponerse a prueba frente a las reconstrucciones históricas racionales que originan, tal como lo plantea por ejemplo Lakatos, descansan sobre lo que ha llamado "intuicionismo epistémico" y que erróneamente identifican "corrección metodológica" con "racionalidad"; según Laudan, tales posiciones están comprometidas con la idea de que lo racional es lo correcto metodológicamente - según la metodología propuesta, por supuesto y viceversa. Laudan sugiere que tanto el intuicionismo como esta identificación entre la corrección metodológica y la racionalidad deberían eliminarse de una satisfactoria filosofia de la ciencia. Por ahora dejaré de lado el problema del intuicionismo en el sentido que plantea Laudan, y me concentraré en el problema de la identificación entre corrección metodológica y racionalidad. Laudan alega que dicha identificación es incorrecta y, más aún, que la noción útil filosóficamente -que entonces debería ser la que promoviera una correcta filosofia de la ciencia- es la de progreso y no la de racionalidad. Por eso, desde su punto de vista, la corrección metodológica debe verse como si condujera al progreso pero no debe identificarse con racionalidad.

Ya he señalado que para ver por qué el nivel de la racionalidad del proceso no puede reducirse a las decisiones de los científicos, es necesario considerar seriamente la idea de que los científicos operan, es decir, toman decisiones, actúan, aceptan y rechazan creencias, erc., con base en un marco conceptual presupuesto, el cual incluye, entre otras cosas, creencias y conocimiento previo, normas y valores, en función de los cuales se fijan las metas de su quehacer científico.

En su análisis de la racionalidad Laudan se limita al sentido de la racionalidad de las decisiones y acciones de los científicos cuando éstas son internas con respecto a un cierto marco conceptual. Pero más aún, para Laudan este sentido de racionalidad queda constreñido a la racionalidad instrumental, a la elección de medios para obteneer determinados fines.

Así, Laudan descuida el sentido de racionalidad del proceso, el cual requiere de la noción de racionalidad completa, y no debe limitarse a la de racionalidad instrumental. Más aún, este sentido exige que se dé cuenta de la transformación de los marcos conceptuales y de cómo ciertos marcos o bien llegan a ser abandonados por completo, o bien llegan a ser transformados completamente. Hemos visto que el meollo de la idea de la racionalidad del proceso del desarrollo científico es que dichas transformaciones y sustituciones de los marcos concepruales son susceptibles de una reconstrucción racional, y por consiguiente, si el proceso de desarrollo científico se entiende como la sucesión y transformación de los marcos conceptuales, entonces la noción de racionalidad instrumental resulta insuficiente para dar cuenta de estas transformaciones. Sostendré, más aún, que la noción de progreso que Laudan propone - si bien es correcta y completamente admisible - contra lo 
que él sugiere, sí presupone una cierta noción de racionalidad. Mostraré que incluso en los propios términos de Laudan su noción de progreso su noción de racionalidad.

Veamos ahora la línea de argumentación de Laudan. Este autor sostiene que la racionalidad es específica al agente y al contexto (agent-and contextspecific), y con base en esta idea concluye que es injusto evaluar la racionalidad de científicos del pasado comparando sus métodos y acciones y decisiones metodológicas tomando nuestros métodos y fines como estándares. Esta última idea es ciertamente correcta. Más aún, Laudan alega que una evaluación de ese estilo es injusta porque pierde de vista que "tanto las metas como las creencias previas de los científicos (background knowledge) varían de agente a agente y que particularmente éste es el caso cuando se habla de épocas muy diferentes de la nuestra" (Laudan, 1987, p. 21).

Que los fines y las creencias previas de los científicos varían de época en época es también ciêrto, sin lugar a dudas. Lo que no es claro es que la pretensión de que una cierta metodología deba reconstruir a la ciencia del pasado o a la historia de la ciencia como racional quiera decir que la racionalidad de los cientificos del pasado deba ser juzgada por comparación con nuestros métodos, estándares $y$ fines. En esto se aprecia que la concepción de racionalidad que se asume en este punto es la de racionalidad instrumental y que es la que suscribe Laudan en su crítica. Esto significa estrechar inadecuadamente la noción de racionalidad (compárese con la de racionalidad completa propuesta arriba) y veremos adelante que esto es lo que le permite separar tan tajantemente como él quiere hacerlo a la "racionalidad" y al "progreso". En efecto, Laudan suscribe una interpretación de la noción de racionalidad según la cual alguien actúa racionalmente si actúa de un modo en el que cree que logrará sus fines. Así, la sugerencia de Laudan es que independientemente de la reconstrucción metodológica que se haga desde el punto de vista del filósofo de la ciencia, muchas de las decisiones de los científicos del pasado y muchos episodios de la historia de la ciencia deben ser juzgados como racionales, ciertamente no porque hayan promovido nuestros fines, sino porque satisfacen las condiciones que prácticamente todo mundo aceptaría como las condiciones mínimas para calificar de racional una decisión o una acción (bajo una concepción de racionalidad instrumental, debemos añadir). Dichas condiciones deben tomar en cuenta lo siguiente:

-las acciones que se realizaron;

-los fines o metas de los agentes;

-los conocimientos previos que sirvieron de base para los juicios acerca de las consecuencias probables de sus posibles acciones. 
Considerando esto, las acciones o las decisiones de los agentes deben considerarse racionales si los agentes actuaron del modo en que creían que promoverían sus fines, con base en la información a su disposición.

Esta concepción de la racionalidad, como ya mencioné antes, es completamente aceptable, mientras no se pretenda que agota todos los sentidos de la noción de racionalidad; y por supuesto tiene un dominio de aplicación legítimo dentro del problema que nos ocupa, si pensamos en las decisiones de los científicos cuando se toman dentro de cierto marco conceptual donde están especificados los valores y fines en función de los cuales se decide o se actúa, así como los recursos disponibles de entre los cuales deben obtenerse los medios para la realización de los fines; el marco conceptual también permite decidir lo que es realizable y por consiguiente lo que es racional perseguir.

Una lectura literal y simplista del pasaje aludido de Laudan conduciría a la conclusión de que en este punto él está atacando molinos de viento, pues ¿quién ha afirmado que el deber de una metodología de la ciencia es exhibir a los científicos del pasado como racionales en el sentido de que sus decisiones deberían promover nuestros fines? Sin embargo, si se toma en cuenta que al menos algunas de las propuestas de la filosofía de la ciencia de corte historicista efectivamente presuponen que es posible identificar (intuitivamente) algunos episodios de la historia de la ciencia como racionales, con independencia de una teoría de la racionalidad, lo cual permite poner a prueba, frente a dichos episodios, una metodología, entonces es posible que en la cidentificación intuitiva" de lo que es racional estén incluidos de contrabando nuestros fines y valores. Ahora bien, si esos episodios identificados como racionales son los que sirven como piedras de toque para juzgar la racionalidad de comunidades pasadas, entonces, a fortiori, se evaluará la racionalidad de otras comunidades por comparación con nuestros fines, valores y creencias previas. (Debo esta observación a Ana Rosa Pérez Ransanz.)

Desde la posición que aquí se defiende, claramente se distingue entre episodios históricos de hecho y las reconstrucciones racionales. Los primeros no son vistos aquí como piedras de toque para evaluar las reconstrucciones racionales, éstas deben ser evaluadas por sus propios méritos por medio de análisis conceptuales, aunque deben ajustarse a los datos históricos, es decir, deben ofrecer evidencia de que los marcos conceptuales reconstruidos efectivamente eran los que estaban disponibles en la(s) época(s) en cuestión. Desde la posición aquí propuesta no surge el problema del "intuicionismo" que Laudan critica.

Con lo señalado hasta aquí puede apreciarse por qué Laudan rechaza la norma metametodológica mencionada al principio (NMF). Su argumento es que los científicos del pasado pueden haber tomado decisiones racionales, $o$ actuado racionalmente, en función de sus fines, valores y creencias previas, y 
no haber promovido nuestros fines, cosa por la cual no deberían ser tachados de irracionales. Esto es correcto, pero como ya he señalado, lo es bajo una concepción de racionalidad instrumental y puede sostenerse sólo bajo el supuesto de que los científicos cuyas decisiones son calificadas de racionales operan bajo un marco dado de fines, valores, normas, y bajo un conjunto dado de creencias y conocimiento disponible, es decir, actúan bajo un cierto marco conceptual y la decisión se refiere a la elección de medios para obtener ciertos fines, todo lo cual es interno al marco conceptual en cuestión.

Conviene acalarar que la presente discusión metametodológica gira en torno al problema de lo que los modelos de desarrollo científico deben proporcionar, y en particular la posibilidad de las reconstrucciones racionales del desarrollo científico. Asimismo, desde el nivel metametodológico se plantea el problema de la comparación y evaluación de metodologías, esto es, de los modelos de desarrollo específicos. Todo esto debe poderse hacer al nivel metametodológico sin tener que asumir ningún compromiso específico con algún modelo en particular, es decir, sin tener que asumir el compromiso con algún conjunto específico de métodos, valores, fines, creencias previas, etc., y más aún, todo esto puede hacerse de una manera que es compatible con la idea de que no existen fines ni valores ni normas, ni reglas metodológicas válidas para todo el proceso de desarrollo científico. He insistido en que ésos son los elementos constitutivos de cada marco conceptual y el proceso de desarrollo científico consiste precisamente en la transformación de esos marcos.

En otros trabajos Laudan ha expresado y defendido correctamente la idea de que el proceso de desarrollo científico involucra lo que aquí se han llamado marcos conceptuales, y que lleva consigo una transformación de creencias sustantivas, de reglas metodológicas y de valores y fines (véase por ejemplo Laudan, 1984), pero debo insistir que lo que está bajo discusión aquí es su tesis en el artículo citado (1987), según el cual para una metodología de la ciencia es irrelevante la posibilidad de reconstruir racionalmente el proceso de desarrollo científico, e igualmente es irrelevante para la metodología la conexión entre la reconstrucción racional y el carácter progresivo del proceso y que lo único importante es la reconstrucción progresiva. Mostraré que incluso en sus propios términos existe una conexión ineludible entre progreso científico y racionalidad.

Pero antes, para reforzar la idea de que el argumento de Laudan se basa únicamente en una noción de racionalidad instrumental y ver algunos de los obstáculos que se le presentan, conviene examinar la defensa que él hace frente a una posible réplica, la cual sigue aproximadamente la línea que sugerí arriba, es decir, la de aplicar el predicado de racionalidad sobre el proceso de desarrollo científico y no sobre decisiones específicas de los científicos dentro de marcos conceptuales particulares. (Aunque no coincide con el planteamiento que aquí he hecho, porque ya he adelantado que éste rechaza la idea de que 
existan fines, valores y reglas metodológicas válidas para todo el proceso de desarrollo científico y para la réplica que contempla Laudan si la supone de ese modo.)

Así pues, Laudan explícitamente considera la réplica que diría que "un agente es racional sólo en la medida en que sus acciones tienden a promover estas 'metas generales' de la ciencia, incluso si sus intenciones (es decir, los objetivos que motivaron sus acciones) fueron muy diferentes de aquellos de la ciencia" (Laudan, 1987, p. 22). Laudan rechaza esto mediante la siguiente observación:

Pero no puedo aceptar la violencia que hace a nuestra noción usual de racionalidad implicando, entre otras cosas, que los agentes que actuaron efectivamente de una manera que promovió sus fines pueden resultar irracionales (a saber, si sus acciones no promovieron los fines de la ciencia), y que agentes tristemente fracasaron en promover sus fines por medio de sus acciones pueden resultar racionales (específicamente cuando sus acciones, de modo inadvertido, promovieron los fines de la ciencia). (Laudan, 1987, p. 23)

Esta afirmación se basa en la idea de que científicos del pasado pueden haber tenido fines diferentes de los nuestros. Nuevamente, es cierto que pueden haber sido racionales (instrumentalmente) al perseguir sus fines mediante ciertos medios a su disposición. Pero esta respuesta señala explícitamente que en el pasado pueden haber existido marcos conceptuales con fines y con creencias previas completamente diferentes a los de un marco conceptual posterior. Entonces surge legítimamente la pregunta de cómo es que los fines y las creencias previas se transformaron y de cuál es la cadena de marcos conceptuales que lleva de un extremo al otro. Esto es, aun en sus términos, surge para Laudan el problema de explicar el proceso de transformación de los marcos conceptuales.

Laudan pasa por alto este problema, es decir, trata explícitamente de la racionalidad instrumental de un agente (o de un grupo de agentes), en cuanto a sus creencias, decisiones o acciones, tomando en cuenta un conjunto dado de fines y de creencias previas, pero no trata la cuestión de las transformaciones y sustituciones de los marcos conceptuales dentro del proceso de desarrollo científico.

Más aún; todavía en sus propios términos Laudan debería enfrentar otro problema que, sin embargo, deja de lado y ante el cual no tendría instrumentos conceptuales adecuados para analizarlo mientras mantenga su noción de racionalidad constreñida a la instrumental. En efecto, Laudan da por hecho que el objetivo fundamental en cada contexto científico es la efectividad, medida de acuerdo con los fines de cada contexto. Pero para que esto funcione se requicre de una concordancia entre las reglas, de acuerdo con el análisis de Laudan, y regularidades empíricas (es decir, entre la regla "si uno quiere 
$y$, entonces debería hacer $x$ " y la regularidad "si se hace $x$, entonces con toda probabilidad $\longrightarrow$ con probabilidad mayor que las otras que se encuentran

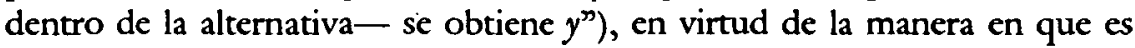
el mundo. Pero no analiza cómo sería posible dentro de una comunidad poner en cuestión el objetivo y llegar a un acuerdo sobre el mismo. La racionalidad completa puede servir para abordar este problema, pero no la racionalidad constreñida a la instrumental que Laudan defiende.

Por otra parte, la objección de Laudan no se aplica a la idea de racionalidad del proceso de desarrollo científico que he defendido arriba, pues según ella, es perfectamente posible que científicos que hayan promovido sus fines (diferentes a los nuestros) y lo hayan conseguido, sean visto como racionales (instrumentalmente), pero que sus decisiones quizá no hayan contribuido a la racionalidad del proceso ni a su progreso, por ejemplo si sus fines son tan diferentes a los nuestros, y a los de cualesquiera otros marcos conceptuales posteriores, que su contribución sea irrelevante dentro de la reconstrucción del desarrollo científico, aunque pueda ser interesante como un episodio de la historia de la ciencia. Por otro lado, la idea aquí defendida permite entender que agentes que hayan fracasado en obtener sus fines y que entonces hayan sido (instrumentalmente) irracionales hayan encontrado en el proceso estrategias de investigación que desde el punto de vista de otro marco posterior (incluyendo el nuestro) sean útiles y puedan haber llevado a la modificación, por ejemplo, de reglas de investigación. Si aquellos agentes pudieran discutir racionalmente con quienes después han valorado así sus hallazgos, podrían ser convencidos de modificar su propio marco conceptual. Por esta razón, un episodio de ese estilo formaría parte de una transformación racional dentro del proceso de desarrollo científico.

Como segunda dificultad de la réplica que discute, Laudan señala que en ésta se asume que en todo momento a lo largo del desarrollo científico existe y persiste el mismo conjunto de fines, el cual es constitutivo de la ciencia. En este sentido Laudan apunta una idea correcta. A lo largo de su historia la ciencia no está constituida por un único conjunto de fines inmutables. En este sentido Laudan presenta una muy acertada crítica a todas las concepciones que consideran que todo el proceso de desarrollo científico está orientado hacia un conjunto único y bien definido de fines, los cuales deberían obtenerse por medio de una única metodología; los fines en cuestión se han planteado de muy diversos modos, destacando entre ellos: la búsqueda o el acercamiento a la verdad, la adecuación empírica y la resolución de problemas.

En el mismo artículo que estamos discutiendo el propio Laudan documenta el tipo de cambio de valores, fines, creencias previas, etc., y en Science and Values (1984) propuso un esquema muy sugerente que ayuda a comprender la estructura de los marcos conceptuales en cada momento determinado de 
la historia de la ciencia. En efecto, Laudan propone concebirlos como compuestos principalmente por creencias sustantivas bien atrincheradas, fines y valores y reglas metodológicas, todo lo cual si bien está interrelacionado, no lo está de manera rígida, es decir, puede haber variaciones dentro de un grupo sin que necesariamente las haya en otro. Ya hemos visto que la noción de racionalidad del proceso de desarrollo científico que aquí se propone asume también esta idea.

Ahora bien, tomando en cuenta la idea de Laudan y lo que antes hemos visto acerca de la estructura de los marcos conceptuales, es posible aplicar a la noción de sucesiones de marcos conceptuales el esquema de internalización y cambio conceptual que ha propuesto Shapere, y así hacer ver que es posible que incluso para los agentes que operan con un cierto marco conceptual hayan existido buenas razones para modificar alguno o algunos de los elementos del marco, desde principios lógicos hasta creencias sustantivas y fines. A diferencia de la mera reconstrucción racional, la aplicación de esta idea nos permitiría analizar los casos concretos en la historia de la ciencia en los que la transformación de un marco conceptual efectivamente ocurrió de manera racional. De este modo también puede explicarse que de hecho existan transformaciones racionales de marcos conceptuales y que en una sucesión de tales marcos, marcos conceptuales que formen parte de la misma línea de desarrollo científico pero que no sean sucesivos puedan no compartir ninguno de los tres conjuntos de elementos básicos: valores y fines, reglas y normas, creencias y conocimientos previos (véanse por ejemplo Shapere, 1986b y 1987).

Pero insistamos en que la transformación de los marcos conceptuales no puede ser juzgada únicamente en términos de racionalidad instrumental, porque tales transformaciones involucran cambios de fines, de valores y de normas, de creencias previas, de concepciones del mundo e incluso de reglas y principios de inferencia.

\section{El progreso implica racionalidad incluso en los términos de Laudan}

Mostraré ahora que incluso si dejamos de lado la idea de que el proceso de transformación de marcos conceptuales involucra una noción de racionalidad mucho más compleja que la de racionalidad instrumental, de acuerdo con sus premisas Laudan queda comprometido con la idea de que el progreso del proceso de desarrollo científico presupone la racionalidad instrumental. Por consiguiente no es posible sostener, como él quiere hacerlo, que la pretensión de que una metodología debe reconstruir a la ciencia del pasado como racional está totalmente equivocada. Por el contrario, de acuerdo con el planteamiento de Laudan, si una metodología reconstruye el proceso de desarrollo como progresivo, a fortiori lo debe reconstruir como racional (aunque de acuerdo con su planteamiento ésta tenga que ser la racionalidad instrumental). 


\section{Para Laudan,}

la metodología no tiene nada que ver con la exhibición o explicación de la racionalidad de científicos del pasado. Lo que requiere de explicación es el hecho de que la ciencia haya sido tan sorprendentemente exitosa en la producción de bienes epistémicos. Nosotros tomamos en serio a la ciencia precisamente porque ha promovido fines que consideramos cognitivamente importantes. Más aún, se ha convertido progresipamente más exirosa conforme pasa el tiempo. Si se pregunta “iexitosa de acuerdo con quién?", o “progresiva de acuerdo con cuáles estándares?”, la respuesta, por supuesto, es: exitosa según nuestros criterios; progresiva de acuerdo con nuestros estándares. (Laudan, 1987, p. 28)

Más adelante Laudan agrega:

a diferencia de la racionalidad, el progreso no tiene que ser una noción específica de los agentes. Podemos hablar sin contradicción, y a menudo lo hacemos, acerca de cierta sucesión de eventos como si representaran un progreso aun cuando los productos finales de esa sucesión estén lejos de lo que los actores involucrados hayan buscado. (Ibid.)

Laudan dice que debe tomarse en cuenta a la historia de la ciencia:

no porque los científicos sean siempre, o más frecuentemente, racionales en comparación con los demás (más bien dudo que lo sean), sino más bien porque la historia de la ciencia - a diferencia de la de otras disciplinas- ofrece un registro impresionante de acciones y decisiones que a través del tiempo se acercan a la realización de fines que la mayoría de nosotros consideramos importantes y que valen la pena. El registro que forma la historia de la ciencia nos muestra cuáles son los tipos de ambiciones cognitivas que se han realizado y cuáles no. $\mathrm{Si}$ ahora abrazáramos objetivos cognoscitivos que no se hubieran realizado progresivamente en el desarrollo de la ciencia entonces esa historia pondría pocos constreñimientos sobre nuestras meditaciones metodológicas. (Ibid.)

Si bien todo esto que dice Laudan en relación con el progreso es aceptable, hace falta explicar, entre otras cosas, cómo ha sido posible tal progreso. La idea que trataré de hacer plausible es que, incluso en los propios términos de Laudan, ese progreso dentro del proceso de desarrollo de la ciencia ha sido posible porque en el pasado se han tomado decisiones racionales.

Notemos en primer lugar que existe una relación entre la racionalidad de acciones y decisiones de científicos del pasado y el carácter progresivo del proceso de desarrollo científico, la cual el mismo Laudan está obligado a aceptar. En efecto, de acuerdo con lo que dice, la historia de la ciencia es un proceso en el cual se van seleccionando fines cognoscitivos y estrategias de investigación. Dicha selección está basada en elecciones y decisiones de científicos 
del pasado. Cuando en el pasado se han ensayado estrategias y se han logrado fines que nosotros valoramos, nosotros encontramos una base que nos permite aceptar esas estrategias que vemos como realizables - ya que de hecho se ha optado por ellas - y podemos constatar que han sido existosas para lograr esos fines que nosotros valoramos. En algunos de esos episodios los científicos del pasado tienen que haber sido racionales: tienen que haber sostenido explícitamente ciertos fines realizables y deben haber seguido estrategias que les permitieron realizarlos. Si sus estrategias no hubieran funcionado para obtener al menos algunos de sus fines, ¿jpor qué habrían sido seleccionadas en el proceso de desarrollo científico? Es cierto que pueden haber tenido ciertos fines que no hayan sido realizados y que correctamente los podamos juzgar (instrumentalmente) irracionales. Pero para que nosotros ahora contemos con evidencia positiva que nos permita decidir que ciertas estrategias son adecuadas de acuerdo con fines que nosostros valoramos, tienen que haberse realizado acciones y tomado decisiones exitosas por parte de los científicos del pasado, las cuales sean pertinentes para nuestros fines; y para darnos cuenta de eso algunos de sus fines deben estar en relación con los nuestros. El propio Laudan da la pista para sostener csto:

Si podemos tener evidencia de que siguiendo cierta regla se promuevan nuestros fines básicos de una manera mejor que si se sigue alguna de sus rivales conocidas, entonces tenemos bases para suscribir la regla. Si tenemos evidencia de que actuar conforme a la regla ha impedido la realización de nuestros fines cognoscitivos, tenemos bases para rechazar la regla. De cualquier modo su estatus queda indeterminado. De esta manera, debería ser posible en principio construir un cuerpo de reglas y procedimientos metodológicos complejo utilizando inicialmente sólo principios de apoyo evidencial simple. (Laudan, 1987, p. 26)

Una dificultad que surge para Laudan en su concepción del papel clave de la historia de la ciencia es que él propone una estrategia que conduciría a la idea de que la metodología que se propone ahora es el resultado de lo que se ha hecho bien en la historia de la ciencia. Pero para que su estrategia pueda funcionar se requiere que a lo largo de la historia exista ya la base de evidencia que nos permita decidir a favor de determinada regla. Pero eso supone que ciertos científicos en el pasado ya hayan hecho selecciones sobre esa base. En ese sentido han sido racionales. Así pues, el funcionamiento de su estrategia presupone que en el pasado ya ha habido científicos que han hecho elecciones racionales. Cierto que esa elección debe haberse hecho en relación con sus fines, pero de acuerdo con lo que el propio Laudan ha expresado, icómo podemos nosotros saber que la estrategia seleccionada funciona para obtener alguno de nuestros fines si ninguno de ellos fue perseguido por científicos del pasado? A fortiori, si tenemos una base para nuestra inducción, es que sabemos que algunas de esas estrategias desarrolladas por científicos del pasado son 
útiles para obtener alguno(s) de nuestros fines. Pero entonces alguno de esos fines debe haber sido perseguido explícitamente, y debe haber sido realizado por los científicos del pasado. Esto es, para tener una garantía de la utilidad de nuestra regla debemos pensar que los científicos del pasado, cuyas decisiones y logros constituyen nuestra base inductiva, tenían (entre otros) el mismo fin, pues de otro modo, de acuerdo con el criterio de Laudan no podemos tener una garantía razonable de nuestra regla.

Esta idea parece reforzarse en el siguiente pasaje:

Ante aquellos que dan por hecho que (como alguna vez lo formuló Janet Kourany) "el objetivo de la metodología es articular" los "criterios de evaluación que de hecho son empleados en la más grande o la más exitosa ciencia", replico que ése no es el objetivo más importante de la metodología; en efecto, si tengo razón, ni siquiera es parte del objetivo de la metodología articular tales criterios. Desde mi punto de vista, el principal objetivo de la empresa metodológica es descubrir las estrategias más efectivas para investigar el mundo natural. (Laudan, 1987, pp. 27-28)

La situación para Laudan, entonces, parece ser la siguiente: una metodología debería descubrir las estretegias más efectivas para investigar el mundo. Pero esto está en función de ciertos fines. Si nuestros fines coinciden con los de científicos del pasado, entonces podemos encontrar evidencia - sea positiva o negativa - que nos permita, racionalmente, aceptar o rechazar estrategias de investigación. En este sentido existe una clara conexión entre la racionalidad de científicos del pasado, nuestra racionalidad - al elegir entre estrategias previamente ensayadas - y el carácter progresivo del proceso. Pues el que sea progresivo y que se hayan seleccionado ciertas estrategias depende de que hayan ensayado exitosamente en el pasado y, por consiguente, que científico del pasado hayan optado racionalmente por determinadas estrategias.

Hasta aquí pretendo haber dado un argumento a favor de que en los términos de Laudan la racionalidad instrumental de los científicos del pasado es necesaria para que nosotros tengamos garantias de que globalmente el proceso de desarrollo científico ha sido progresivo, es decir, que podemos ahora aprovechar estrategias que se ensayaron en el pasado y que sabemos que sirven para promover nuestros fines. La cuestión es que para saber que promueven nuestros fines esos fines deben haber estado presentes en los marcos conceptuales de los científicos del pasado y las estrategias en cuestión deben haberse desarrollado para conseguirlos. De otra manera no tenemos base para nuestra inducción. Esto es, de otra manera tendríamos que empezar de nuevo a ensayar las diferentes estrategias de investigación que nos han sido heredadas, pues no sabríamos cómo funcionan en relación con los fines que nosostros valoramos. 
En conclusión, la pretensión de Laudan de que "el requisito de que una metodología o una epistemología debe exhibir a la ciencia pasada como racional está totalmente equivocado", está equivocada. Su tesis no es sostenible ni siquiera sobre la base de su concepción de la racionalidad que la reduce a la racionalidad instrumental centrada en las decisiones de los agentes.

\section{Conclusiones}

Hemos visto que si adoptamos una noción amplia de racionalidad que incluya tanto a la racionalidad instrumental como a la evaluativa, la ontológica, la epistemológica, la metodológica y la lógica, la cual nos permitiría concebir los procesos de cambio de creencias, reglas, valores y fines con base en razones, entonces la noción de progreso implica la de cambio racional, no sólo en el sentido de que se requiere un comportamiento racional (instrumental) de científicos del pasado para que desde el punto de vista de ahora exista una base de inducción que vuelva razonables las decisiones que ahora se toman y tenga sentido así la noción misma de que ha habido progreso, sino también en el sentido de que ha habido una selección de todos esos elementos que permite la elección de fines realizables, la elección de mejores medios para obtenerlos, la selección de normas y reglas que mejor permiten lograr esos fines, etc.

Pero si además aceptamos que lo anterior admite una reconstrucción por medio de diversos y sucesivos marcos conceptuales, entonces a la luz de cada uno de ellos es posible reconstruir las razones que convencerían a sujetos que se adhirieran a otros marcos conceptuales previos de la conveniencia de aceptar ciertas transformaciones en ellos, transformaciones que incluirian fines y valores, tanto como reglas metodológicas y conocimientos. En este caso, puede apreciarse que la pretensión de que el último marco dentro de la sucesión ha resultado y se ha beneficiado del desarrollo y de las transformaciones de los anteriores, es decir, la pretensión de que el desarrollo ha sido progresivo, puede explicarse porque el proceso ha sido racional, esto es, desde cada marco posterior es posible encontrar razones para modificar al marco previo, y tal reconstrucción racional no depende de que de hecho hayan ocurrido los episodios históricos en los cuales los científicos de determinada comunidad hayan hecho elecciones racionales. La racionalidad del proceso es autónoma e irreducible a la racionalidad de los científicos individuales y sus comunidades.

En suma, la noción de que el proceso de desarrollo científico ha sido progresivo exige explicaciones de los pasos de un marco conceptual a otro. Dentro de esta explicaciones debe tomarse en cuenta a los factores epistémicos y a los no epistémicos. En muchas ocasiones el papel de los factores epistémicos es notable y ofrece una explicación de un proceso que se llevó a cabo por la operación de razones para las comunidades en cuestión, o de decisiones y acciones racionales. En otras ocasiones además de factores epistémicos pueden 
haber operado muchos otros, no epistémicos, que el bistoriador de la ciencia debe analizar. Pero cuando se reconstruye como progresiva una sucesión de marcos conceptuales, una de las mejores explicaciones de ese progreso es en términos de la racionalidad del mismo. Con base en lo anterior podemos concluir:

a) La afirmación de Laudan de que la racionalidad no se requiere para dar cuenta del progreso científico está equivocada.

b) La norma metametodológica fundamental expresada al principio es correcta.

c) La aceptación de NMF no nos compromete con una identificación entre "racionalidad" y "corrección metodológica", pues por metodología no se entiende aquí un conjunto de normas y reglas universales que sean aplicables en todas circunstancias. Cierto que la reconstrucción racional presupone una caracterización de la racionalidad, pero la metodología, y por consiguiente la corrección metodológica, no es idéntica a la racionalidad, sino que la metodología presupone la racionalidad.

d) Bajo esta perspectiva la historia de la ciencia no es la piedra de toque para las reconstrucciones racionales y, por consiguiente, no se asumen compromisos previos a una caracterización de la racionalidad.

e) El criterio de elección entre metodologías debe ser modificado al efecto de que reconozca la diferencia entre reconstrucción racional del proceso de desarrollo científico e historia de la ciencia. La modificación puede ser la siguiente:

\section{(CEM') Criterio de elección entre metodologias:}

Una metodología de la ciencia es preferible a otra si ofrece una sucesión de marcos conceptuales cada uno de los cuales permite ofrecer razones aceptables y suficientes para que sujetos racionales que, partiendo del marco previo en una discusión racional, llegaran a modificar su marco de partida y a aceptar el marco sucesivo en virtud de esas razones. Es preferible la metodología que explique mejor, es decir, la que reconstruya más clara y completamente los marcos conceptuales que históricamente existieron, de manera que se expongan claramente las razones que hubieran sido aceptables para quienes partieran del marco previo; esto explica el hecho de que el desarrollo de la ciencia haya alcanzado los niveles actuales de una manera racional y tal que permite asegurar que ha habido progreso en ese desarrollo.

Ante la posible acusación de que este criterio promueve la historia ficción de la ciencia, la réplica es que los marcos conceptuales que la metodología 
debe reconstruir son los marcos conceptuales que históricamente han existido de hecho; no hay nada de ficción en eso. Lo que es cierto es que muchas veces ha habido transformaciones de marcos conceptuales causadas por factores no epistémicos. La reconstrucción racional no toma en cuenta esto, sin que ello implique que eso no sea importantísimo para la historia de la ciencia, la cual debe dar las explicaciones completas de lo que realmente ocurrió y de cuáles fueron sus causas. Esta diferencia, y la idealización que supone la reconstrucción racional (por la eliminación de factores no epistémicos), es la que produce el "aire” de ficción. Pero la ficción no es tal, se trata de la profunda diferencia epistemológica entre el nivel de la metodologla de la ciencia y el de la historia de la ciencia. El nivel de la metodología, entendida como la reconstrucción racional de la ciencia, tiene un estatus epistémico por derecho propio, el cual está relacionado con el de la historia de la ciencia, pero no es el mismo.

El nivel de la metodología es precisamente en donde se deben construir modelos que hagan explícita la racionalidad de la ciencia y de su desarrollo. La racionalidad y el desarrollo de la ciencia son hechos históricos, la disciplina que se encarga de ofrecernos explicaciones de cómo son ellos posibles es la filosofía de la ciencia al nivel de las metodologías. En general, la epistemología parte del hecho de que existe el conocimiento y una de sus tareas es decirnos cómo es el conocimiento posible, cómo se justifica y cómo se desarrolla. La filosofía de la ciencia, al nivel de la metodología, parte del hecho de que hay conocimiento científico, su tarea es la de explicarnos cómo se desarrolla. Lo que hasta ahora nos ha enseñado es que se desarrolla por medio de una sucesión de marcos conceptuales, sucesión que es progresiva porque es racional. Cierto que existe una fuerte competencia entre diferentes metodologías para detallar el mejor modelo. Arriba hemos ofrecido un criterio para elegir entre los diversos modelos que ahora se nos ofrecen. Una buena razón para rechazar ciertos modelos - como el que Laudan sugiere en su trabajo de 1987es que sólo describen el carácter progresivo del proceso de desarrollo de la ciencia, pero no explican por qué lo es.

La historia de la ciencia no puede ser entonces la piedra de toque para decidir y evaluar metodologías, pero en la medida en que los marcos conceptuales reconstruidos por cada metodología deben ser los que históricamente existieron, la historia de la ciencia ejerce un importante control sobre la metodología. El nivel de las metodologías no es una disciplina empírica, ni cada metodología es un modelo de aspectos de la realidad empírica. El nivel de las metodologías forma parte de una disciplina filosófica encargada de hacer análisis conceptuales. Por eso no debe extrañar que no exista una piedra de toque empírica para su contrastación. La evaluación de las metodologías, tratándose de uno de los niveles de la filosofía de la ciencia, no puede ser sino 
con base en análisis conceptuales y en razonamientos, controlados, eso sí, por los datos de la historia conceptual de la ciencia.

\section{BIBLIOGRAFIA}

Bunge, Mario, 1987, “Seven Desiderata for Rationality”, en J. Agassi y I. C. Jarvie (comps.), Rationality: The Critical View, Martinus Nijhoff Publishers, Dordrecht.

Shapere, Dudley, 1986b, “Objectivity, Rationality and Scientific Change", en P. Kitcher y P. Asquith (eds.), PSA 1984, vol. II, .

_- 1987, "Method in the Philosophy of Science and Epistemology: How to Inquire abour Inquiry and Knowledge", en N. Nersessian (comp.), The Processes of Science, Martinus Nijhoff Publishers, Dordrecht.

Olivé, León, 1987, "Two Conceptions of Truth and their Relationships to Social Theory", Philosophy of the Social Sciences, vol. 17, no. 3, septiembre de 1987.

Lakatos, Imre, 1987, "History of Science and its Rationals Reconstructions", en The Methology of Scientific Research Programmes, Philosophical Papers I, Cambridge University Press.

Laudan, Larry, 1984, Science and Values, University of California Press.

—, 1986, "Intuitionist Meta-Methodologies”, Synthese, vol. 67, pp. 115-129.

—, 1987, "Progress or Rationality? The Prospects for Normative Naturalism", American Philosophical Quarterly, vol. 24, no. 1, enero de 1987, pp. 19-31.

Wykstra, 1980, "Toward a Historical Meta-Method for Assessing Normative Methodologies: Rationability, Serendipity, and the Robinson Crusoe Fallacy", PSA 1980, vol. 1, pp. 211-222. 